Check for updates

Cite this: RSC Adv., 2017, 7, 41043

Received 27th June 2017

Accepted 10th August 2017

DOI: $10.1039 / c 7 r a 07109 d$

rsc.li/rsc-advances

\section{Variable self-assembly and in situ host-guest reaction of beta-cyclodextrin-modified graphene oxide composite Langmuir films with azobenzene compounds $\dagger$}

\author{
Yagui Gao, ${ }^{\text {ab }}$ Tifeng Jiao, (DD *ab Kai Ma, ${ }^{\text {b }}$ Ruirui Xing, ${ }^{\text {bc }}$ Lexin Zhang, ${ }^{\mathrm{b}}$ Jingxin Zhou*b \\ and Qiuming Peng ${ }^{\mathrm{a}}$
}

\begin{abstract}
The preparation of graphene oxide (GO)-based composite Langmuir films play important roles in the application of self-assembly nanomaterials. In this work, beta-cyclodextrin-modified graphene oxide (GO-CD) and the azobenzene-modified PAA derivative (abbreviated as PAA-Azo) have been designed and synthesized. The interfacial assemblies and host-guest interactions of GO-CD material with used two azobenzene compounds (N-Azo and PAA-AzO) in the obtained Langmuir and Langmuir-Blodgett (LB) films were investigated. All the spreading films from different subphases were transferred onto solid substrates and their nanoscale morphologies as well as self-assembled characteristics were investigated by some methods including atomic force microscopy, UV-vis spectra, Raman spectra, etc. The obtained results indicated that there existed host-guest interaction between beta-CD segments with azobenzene groups. Besides, in the obtained GO-CD/N-Azo films, there also appeared J-aggregate of N-Azo molecules on $G O$ sheet surface. However, there were only host-guest interactions without any aggregates of azobenzene segment in the GO-CD/PAA-Azo composite films. Hence, the presently obtained GO-CD/azobenzene composite Langmuir films provide new clues to develop GO-based selfassembled films as well as nanomaterials towards nano-device and sensor applications.
\end{abstract}

\section{Introduction}

Graphene oxide (GO)-based composites have been shown to be particularly attractive over recent years, owing to their special electronic, mechanical, and thermal properties. ${ }^{1-3}$ In recent years, more attention has been devoted to reasonable design and targeted synthesis of various functionalized GO-based nanocomposites, demonstrating better processability and adaptation for nanomaterial applications. ${ }^{4-6}$ The functionalized modification of GO materials with organic groups are needed for the purposes of good dispersion and variable applications. ${ }^{7-9}$ For example, porphyrin, dopamine, isocyanate derivatives, long-chain alkylamine or tetrathiafulvalene, have been used to decorate GO to obtain a well dispersed state in solvents for designed properties. ${ }^{10-14}$ Compared with the above used small

${ }^{a}$ State Key Laboratory of Metastable Materials Science and Technology, Yanshan University, Qinhuangdao 066004, P. R. China. E-mail: tfiao@ysu.edu.cn

${ }^{b}$ Hebei Key Laboratory of Applied Chemistry, School of Environmental and Chemical Engineering, Yanshan University, Qinhuangdao 066004, P. R. China. E-mail: zhoujingxin@ysu.edu.cn

'State Key Laboratory of Biochemical Engineering, Institute of Process Engineering, Chinese Academy of Sciences, Beijing 100190, P. R. China

$\dagger$ Electronic supplementary information (ESI) available. See DOI: $10.1039 / \mathrm{c} 7 \mathrm{ra} 07109 \mathrm{~d}$ molecules, some polymers, such as poly(2-(dimethylamino) ethyl methacrylate) and poly(vinyl alcohol), can be also employed to modify GO via different chemical reactions to enhance the properties of GO-based composites in obvious domains. ${ }^{15,16}$ However, some problems still appears, such as incomplete adsorption of organic molecules, aggregation between GO sheets, and undesired side effects. On the other hand, host-guest chemistry of cyclodextrin has drawn important development in recent years owing to easy modification, high selectivity and yields. ${ }^{17-20}$ For example, Li and coworkers have achieved numerous works about design and application of beta-CD functionalized carbon materials in guest enrichment or sensors. ${ }^{21-28}$ It is reported that the a host-guest reaction has been displayed to functionalize GO with cyclodextrin-based [2] rotaxane array for the development of GO-based photoswitches and cyclodextrin functionalized graphene-gold nanoparticle hybrids for electrochemical thrombin aptasensor. ${ }^{29,30}$ Therefore, it seems an interesting challenge to directly prepare GO-based composites with special host-guest reaction in a selfassembled systems.

On the other hand, Langmuir and Langmuir-Blodgett (LB) techniques can be regarded as effective techniques to synthesize interfacial thin films. ${ }^{31-38}$ Since the initial reports from Huang's group about GO self-assembly process in Langmuir 
films,${ }^{39,40}$ several studies show the preparation of GO monolayer or GO-based composite films via LB assembly method. ${ }^{41-47} \mathrm{Up}$ to now, the inclusion complex formation of azobenzenecontaining compounds and beta-cyclodextrin (beta-CD)/alphacyclodextrin at the air/water interface was investigated and characterized. ${ }^{4-52}$ It can be interesting that a combination of GO-based composites self-assembly films involved in hostguest interactions via beta-CD and azobenzene segments by Langmuir technique should be particularly advantageous due to excellent molecular recognition, moderate self-assembled nanostructures, and designed self-assembly models. Thus, aqueous solutions of two soluble azobenzene-containing compounds (abbreviated as N-Azo and PAA-Azo) as subphase solutions have been designed and chosen for the preparation of composite films.

In this article, we report the preparation of stable GO-CD composite Langmuir films by interfacial host-guest interactions with azobenzene compounds in subphase solutions in a facile and effective manner. We discover that self-assembly and host-guest interactions between beta-CD groups modified GO sheets and soluble azobenzene-containing small molecules or polymeric molecules are responsible for formation of present composite films. Also, the as-prepared Langmuir films can be easily transferred onto solid substrates via Langmuir-Blodgett (LB) assembly technique and characterized by morphological and spectral methods. Thus, the present GO-based composite Langmuir films will provide significant potential applications in nano-device and biosensor design.

\section{Materials and methods}

\subsection{Materials}

The experimental used materials, beta-cyclodextrin (98\%, abbreviated as beta-CD), ethylenediamine (99.5\%), chloroacetic acid, 4-aminoazobenzene (abbreviated as N-Azo), and poly(acrylic acid) (abbreviated as PAA, M.W. $~ 450000$ ) were purchased from Aladdin Reagent (Shanghai, China), Alfa Aesar (Tianjin, China) Chemicals as well as TCI Shanghai Chemicals, and used without further purification. Graphite powder (325 mesh, 99\%) was obtained from Alfa Aesar Chemicals (Shanghai, China). $\quad N$-(3-Dimethylaminopropyl)- $N^{\prime}$-ethylcarbodiimide hydrochloride (EDC-HCl) and $N$-hydroxysuccinimide (NHS) were obtained from Sigma-Aldrich without any purification. Other reagents were purchased from Beijing Chemicals and used without any purification. All used water was purified in a double-stage Millipore Milli-Q Plus purification system. Firstly, graphene oxide (GO) was synthesized from graphite powder by a modified Hummers method. ${ }^{53}$ Then, the carboxyl-modified $\mathrm{GO}$ (GO-COOH) was synthesized according to the reported literature, ${ }^{\mathbf{5 4}, 55}$ and lyophilized at a temperature of $-50{ }^{\circ} \mathrm{C}$. Amine-modified beta-CD derivative (abbreviated as $\mathrm{NH}_{2}$-beta-CD) were synthesized according to the literature reports, ${ }^{56,57}$ and the molecular structure (Fig. S1 $\dagger$ ) and purity was confirmed by ${ }^{1} \mathrm{H}$ NMR and ${ }^{13} \mathrm{C}$ NMR (Fig. S2 and S3†) as well as MS (Fig. S4 $\dagger$ ). Beta-CD functionalization on $\mathrm{GO}-\mathrm{COOH}$ was finished following analogous dehydration reaction procedure described in the literatures, ${ }^{58,59}$ and modified in similar procedure. By filtration and dialysis process, the resulting betaCD-modified GO solid (GO-CD) was obtained by freeze-drying. The azobenzene-modified PAA derivative (abbreviated as PAA-Azo) was prepared as described in the following dehydration reaction. The $100 \mathrm{~mL}$ aqueous PAA solution $\left(10 \mathrm{mg} \mathrm{mL}^{-1}\right)$ was mixed with NHS $(3.4 \mathrm{~g})$ and EDC-HCl $(5.7 \mathrm{~g})$ into a roundflask under ice-bath. After stirring vigorously for $2 \mathrm{~h}, \mathrm{~N}-\mathrm{Azo}$ (137 $\mathrm{mg}, \mathrm{ca}$. $5 \mathrm{~mol} \%$ of acrylic acid unit) was added to the mixture and left stirring overnight under ice-bath and next 3 days at room temperature. After that the solid was dispersed with water and dialyzed in water for 4-5 days with dialysis tubing (MWCO 12 400). After dialysis, PAA-Azo powder was obtained by freeze-drying. The characteristic molecular structure was confirmed by XRD and IR spectra (Fig. S9 and S10 $\dagger$ ) as well as elemental analysis (yield 78\%, found: C, 48.21; H, 6.15; N, 6.52; calculated PAA, $\left.\left(\mathrm{C}_{3} \mathrm{H}_{4} \mathrm{O}_{2}\right)_{n}, \mathrm{C}, 50.00 ; \mathrm{H}, 5.59 ; \mathrm{O}, 44.40\right)$.

\subsection{Procedure of $\mathrm{LB}$ films preparation}

The interfacial preparation and LB films transfer were finished via KSV-NIMA Mini-trough LB system. The trough was carefully cleaned with chloroform and ethanol, and then filled with DI pure water, aqueous $\mathrm{N}$-Azo solution $\left(50 \mathrm{mg} \mathrm{L}^{-1}\right)$, or PAA-Azo solution (25 $\left.\mathrm{mg} \mathrm{L}^{-1}\right)$, respectively. After sonication $20 \mathrm{~min}$, GO-CD dispersed solution $\left(0.500 \mathrm{mg} \mathrm{mL}^{-1}\right)$ with different spread solvents was dropwise spread onto the water surface using a glass syringe. Surface pressure was monitored using a tensiometer attached to a Wilhelmy plate. The film was compressed by barriers at a speed of $10 \mathrm{~cm}^{2} \mathrm{~min}^{-1}$ at room temperature. The prepared GO-CD monolayer was transferred to solid substrates at suitable pressure points by vertically pulling method with speed of $2 \mathrm{~mm} \mathrm{~min}^{-1}$. Fresh cleaved mica, glass plates, quartz, and $\mathrm{CaF}_{2}$ were used as the substrates to transfer monolayer or multilayer for next morphological and spectral characterizations. Quartz and glass plates were treated with $1: 1: 5 \mathrm{NH}_{4} \mathrm{OH}: \mathrm{H}_{2} \mathrm{O}_{2}: \mathrm{H}_{2} \mathrm{O}$ (by volume) and washed repeatedly with deionized water before use.

\subsection{Characterization}

The morphology of composite Langmuir films were characterized using a field-emission scanning electron microscope (SEM) (S-4800II, Hitachi, Japan) as well as a transmission electron microscope (TEM) (HT7700, Hitachi High-Technologies Corporation, Japan). Atomic force microscopy (AFM) pictures were measured with a Nanoscope model Multimode 8 Scanning Probe Microscope (Veeco Instrument, USA) with silicon cantilever probes using the tapping mode. UV-vis spectra were recorded with a Shimadzu UV-2550 system (Shimadzu Corporation, Japan). FT-IR spectra were obtained using a Fourier infrared spectroscopy (Thermo Nicolet Corporation) by the conventional $\mathrm{KBr}$ disk tablet method or composite films on $\mathrm{CaF}_{2}$ plates. Raman spectroscopy was measured by a Horiba Jobin Yvon Xplora PLUS confocal Raman microscope equipped with a motorized sample stage. The intensity of a Raman peak was extracted from the maximum value after baseline subtraction over corresponding spectral range. X-ray photoelectron spectroscopy (XPS) was measured by the Thermo Scientific 
ESCALab 250Xi using $200 \mathrm{~W}$ monochromated Al Ká radiation. Both survey scans and individual high-resolution scans for $\mathrm{N}(1 \mathrm{~s}), \mathrm{O}(1 \mathrm{~s})$ and $\mathrm{C}(1 \mathrm{~s})$ peaks were recorded. X-ray diffraction study was carried out by using an X-ray diffractometer (SMART $\mathrm{LAB}$, Rigaku) equipped with a conventional $\mathrm{Cu} \mathrm{K} \alpha \mathrm{X}$-ray radiation $(\lambda=1.54 \AA)$ source and a Bragg diffraction setup. Circular dichroism (CD) spectra of the transferred LB films were recorded on a JASCO J-810 CD spectrometer. In the process of measuring CD spectra, the multilayer film was placed perpendicular to the light path and rotated within the film plane to avoid polarization-dependent reflections and eliminate the possible angle dependence of the CD signals. ${ }^{60-65}$ Elemental analysis was carried out with a Flash EA Carlo-Erba-1106 Thermo-Quest. ${ }^{1} \mathrm{H}$ NMR and ${ }^{13} \mathrm{C}$ NMR spectra were obtained on an ARX400 (Bruker, Fällanden, Switzerland) NMR spectrometer in $\mathrm{D}_{2} \mathrm{O}$ or d-DMSO with TMS as an internal standard. MALDI-TOF mass spectra (MS) were determined with BIFLEXIII in methanol solution. A UV lamp with wavelength of $365 \mathrm{~nm}$ (LUYOR-3405, $20 \mathrm{~mW} \mathrm{~cm} \mathrm{~cm}^{-2}$, LUYOR Corporation) was utilized to irradiate the obtained solutions and LB films to induce the photoisomerization reactions.

\section{Results and discussion}

The obtained beta-CD-functionalized GO-CD material can act as building blocks to prepare Langmuir films via interfacial host-guest interactions with azobenzene-containing molecules by standard Langmuir self-assembly technique. For this, the prepared GO precursor, the intermediates $\mathrm{GO}-\mathrm{COOH}$ and $\mathrm{NH}_{2}$-beta-CD, and the targeted building block GO-CD were synthesized and characterized by many methods. Firstly, the SEM and TEM images of the obtained GO (Fig. S5a and bi) show well-dispersed ultrathin GO sheet with micrometer scale size. In addition, after modification with beta-CD groups, the SEM images of as-prepared GO-CD powder (Fig. S5c and $\mathrm{d} \dagger$ ) demonstrate aggregated state of GO sheet due to possible hydrogen bonding between modified CD segments. And XRD curves of the as-prepared materials show the disappearance of Bragg peaks at $2 \theta$ value of $11.3^{\circ}$ owing to the (001) diffraction peak and presence of new broad peak at $2 \theta$ value of $19.8^{\circ}$ for GO-CD (Fig. S6 $\dagger$ ). Moreover, the FT-IR spectra of the asprepared GO-CD powder and original materials were measured and shown in Fig. S7. $\dagger$ It can be easily observed that the characteristic peak of GO sheet appeared at $3424 \mathrm{~cm}^{-1}$ can be attributed to the $-\mathrm{OH}$ vibration stretching, while other obvious bands demonstrated carboxyl group at $1724 \mathrm{~cm}^{-1}$, epoxy group at $1226 \mathrm{~cm}^{-1}$, and alkoxy group at $1050 \mathrm{~cm}^{-1}$, respectively. ${ }^{66-70}$ In addition, vibrational peaks of $\mathrm{NH}_{2}$-beta-CD at $2927 \mathrm{~cm}^{-1}$ can be assigned to $\mathrm{C}-\mathrm{H}$ vibration stretching, with other peaks at 946 and $756 \mathrm{~cm}^{-1}$ for pyranose ring in CD components. Simultaneously, characteristic peaks corresponding to amide-carbonyl (NH-CO) stretching vibration at $1650 \mathrm{~cm}^{-1}$, $\mathrm{C}-\mathrm{N}$ stretching vibration at $1384 \mathrm{~cm}^{-1}$ suggest that the $\mathrm{GO}-\mathrm{COOH}$ material was modified with $\mathrm{NH}_{2}$-beta-CD via amide bond. Thus, the obtained FT-IR data indicate successful modification of betaCD groups in GO sheet and possible 3D structural composite formation due to chemical functionalization. ${ }^{59}$ The elemental analysis data (Table 1) of as-prepared GO-modified materials demonstrate obvious increment of hydrogen composition and appearance of nitrogen component with value of $3.91 \mathrm{wt} \%$ for obtained GO-CD material. The comparison of XPS data and elemental analysis data show similar calculated N/C ratios, which is almost in agreement with the above obtained results. Thus, we characterized the interfacial phase behaviors and the transferred GO-CD composite LB films by means of morphological and spectral methods.

For the interfacial self-assembly, firstly, we investigated the surface pressure-area isotherms of as-obtained Langmuir films at room temperature. The optimization of used different spreading solvents and volumes for present GO-CD Langmuir film on pure water surface was demonstrated in Fig. S11. $\dagger$ It indicates that the optimized spread condition (methanol/ chloroform $3: 2(\mathrm{v} / \mathrm{v}), 300 \mu \mathrm{L})$ seem suitable for material dispersion on surface and used in next interfacial characterization process. It can be easily observed from Fig. 1 that the isotherm of GO-CD spread on pure water surface with optimized solvent ratio and spread volume show slow increment of pressure at trough area of $125 \mathrm{~cm}^{2}$ until reaching $27 \mathrm{mN} \mathrm{m}^{-1}$ at compression end of $21 \mathrm{~cm}^{2}$. For the GO-CD film on N-Azo subphase, the isotherm show obvious increment of surface pressure from initial compression step and the final pressure reached above $25 \mathrm{mN} \mathrm{m}^{-1}$. In addition, for the GO-CD/PAA-Azo composite Langmuir film, a little increment appear for the initial pressure point at $136 \mathrm{~cm}^{2}$ and final pressure of $28 \mathrm{mN} \mathrm{m}^{-1}$ compared the film from pure water surface. The obvious change in all isotherms suggested the occurrence of interfacial interaction between beta-CD groups in GO-CD building blocks and N-Azo/PAA-Azo molecules in subphase solutions and formation of composite Langmuir films.

The nanoscale morphologies of monolayer GO-CD Langmuir films were investigated by AFM (Fig. 2). The morphological difference is obviously observed. The overlapped GO sheet is representing the GO-CD Langmuir films with the height distribution of $1.1 \pm 0.2 \mathrm{~nm}$ (Fig. 2a), while dispersed packed films with little wrinkles dominate rather than dispersed GO sheets in the case of composite films from N-Azo subphase with the height distribution of $1.6 \pm 0.3 \mathrm{~nm}$ (Fig. 2b). As for the morphology of GO-CD/PAA composite film, messy reticular nanoscale aggregates appear in Fig. 2c. In addition, SEM and TEM images of GO-CD composite LB films from N-Azo and PAA-Azo subphase were also characterized. The SEM images in Fig. $3 \mathrm{~b}$ and d clearly demonstrate distinct dispersed or crosslinked aggregate states after interaction with different azobenzene-containing molecules. The TEM images in Fig. 3e and $f$ also verify the formation of large aggregation in the obtained films. The difference of morphology and height between

Table 1 Elemental analysis data of as-prepared GO-based materials

\begin{tabular}{llll}
\hline Sample & $\mathrm{C}[\mathrm{wt} \%]$ & $\mathrm{H}[\mathrm{wt} \%]$ & $\mathrm{N}[\mathrm{wt} \%]$ \\
\hline GO & $44.30 \pm 0.02$ & $2.01 \pm 0.08$ & - \\
GO-COOH & $44.70 \pm 0.06$ & $2.10 \pm 0.07$ & - \\
GO-CD & $42.84 \pm 0.06$ & $4.79 \pm 0.05$ & $3.91 \pm 0.01$
\end{tabular}




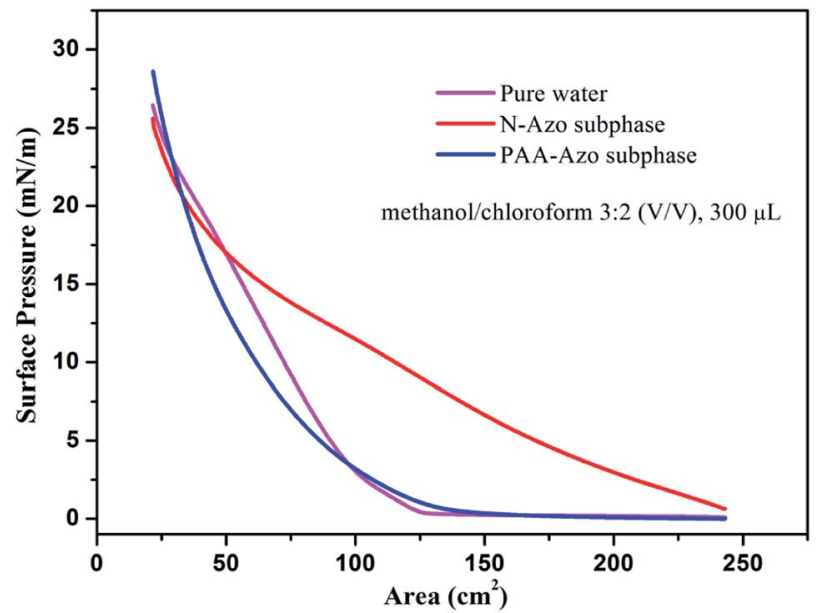

Fig. 1 Surface pressure-area isotherms of Langmuir films of asprepared GO-CD solution (methanol/chloroform v/v 3:2, $300 \mu \mathrm{L}$, $0.5 \mathrm{mg} \mathrm{mL}^{-1}$ ) spread on pure water surface, N-Azo, and PAA-Azo subphases at room temperature.

the GO-CD Langmuir films from different subphase solutions can be mainly owing to the introduction of N-Azo or PAA-Azo molecules in the composite Langmuir film after host-guest interaction.

Azobenzene compounds have characteristic UV-vis absorption. We utilized the UV-vis spectra to investigate the obtained azobenzene derivatives and the multilayer LB films deposited on solid substrates. Fig. 4 shows the UV-vis spectra of the multilayer GO-CD/N-Azo and GO-CD/PAA-Azo composite LB films. At the same time, as shown in Fig. $\mathrm{S} 8, \uparrow$ the GO aqueous dispersed solution displays a peak at $226 \mathrm{~nm}$ which is due to the $\pi-\pi^{*}$ transition of aromatic $\mathrm{C}=\mathrm{C}$ bonds and a shoulder at 290-300 nm which corresponds to the $n-\pi *$ transition of the $\mathrm{C}=\mathrm{O}$ bonds. $^{71-73}$ Thus, the UV-vis spectrum of the GO-CD solution showed strong absorption peak at $282 \mathrm{~nm}$, which could be reasonably ascribed to the charge transfer band of GO sheet with beta-CD segment. In addition, the obtained UV-vis

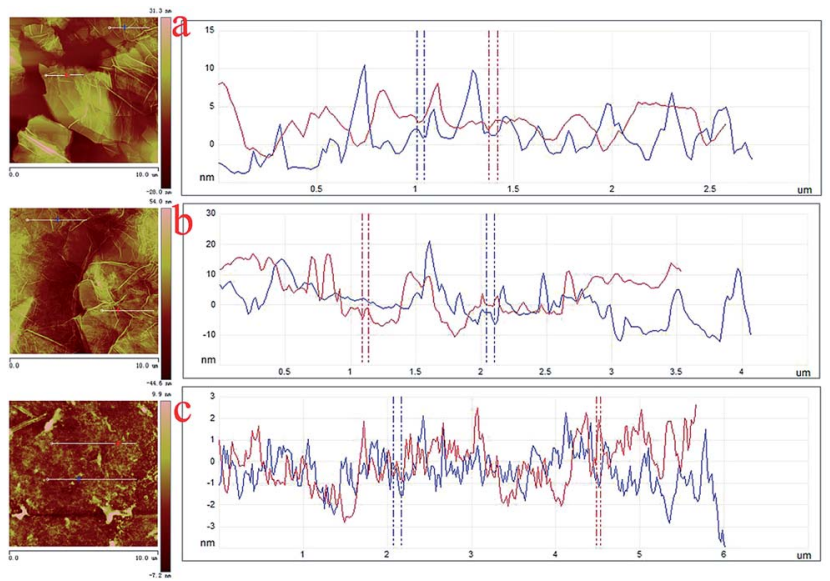

Fig. 2 AFM images with section analysis of monolayer Langmuir films of GO-CD spread on different subphases transferred at $15 \mathrm{mN} \mathrm{m}^{-1}$. (a), pure water surface; (b), N-Azo subphase; (c), PAA-Azo subphase.

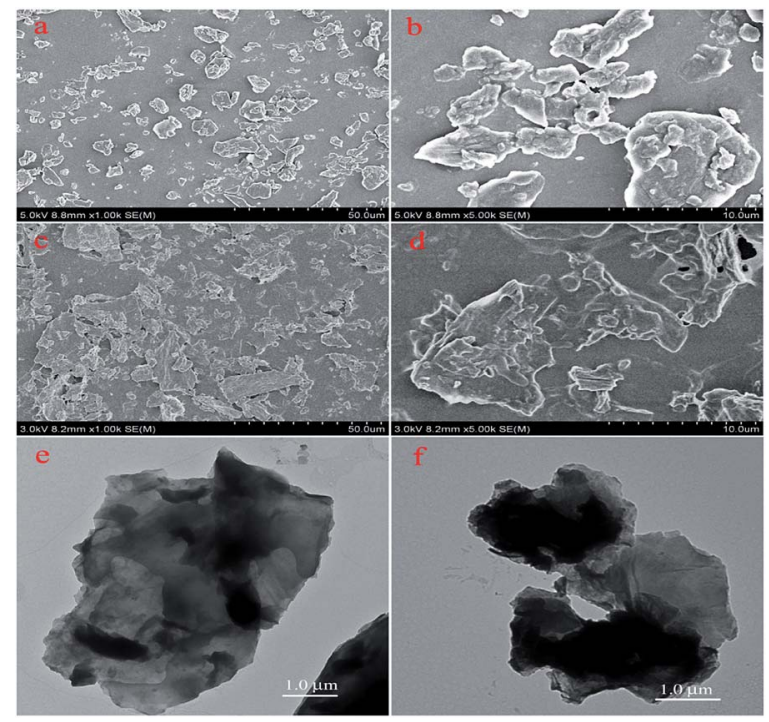

Fig. 3 SEM of 10-layered LB films and TEM images of monolayer LB films of GO-CD from N-Azo subphase ( $a, b$ and e) and PAA-Azo subphase (c, $d$ and f) transferred at $15 \mathrm{mN} \mathrm{m}^{-1}$.

spectrum of aqueous N-Azo solution showed two strong absorption peaks at 230 and $376 \mathrm{~nm}$ and a weak one at $442 \mathrm{~nm}$. The former band can be ascribed to the localized absorption of the aromatic rings, while the peak at $376 \mathrm{~nm}$ and the weak peak at $442 \mathrm{~nm}$ can be assigned to the $\pi-\pi^{*}$ transition of trans isomers and $\mathrm{n}-\pi^{*}$ transition of the cis-isomers, respectively. ${ }^{74}$ When the GO-CD film was deposited from N-Azo subphase, a great change in the absorption spectra was observed. The charge transfer band at $282 \mathrm{~nm}$ disappeared completely, which suggested the decrement of charge transfer between GO sheet with beta-CD segment as well as possible production of hostguest interaction between beta-CD segment and azobenzene part. Simultaneously, the red shift of the maximum absorption of azo group (from $376 \mathrm{~nm}$ to $393 \mathrm{~nm}$ ) indicated the possible formation of J-aggregate of conjugated azobenzene segment in the composite films compared with monomers in the N-Azo solution. In addition, for the GO-CD/PAA-Azo composite films shown in Fig. $4 \mathrm{~b}$, the characteristic band appears at the position of $344 \mathrm{~nm}$ with the same value of PAA-Azo solution. This suggested most of azobenzene segment kept monomers state in the GO-CD/PAA-Azo composite films. In addition, the UV-vis spectra of aqueous mixed solutions of GO/GO-CD with
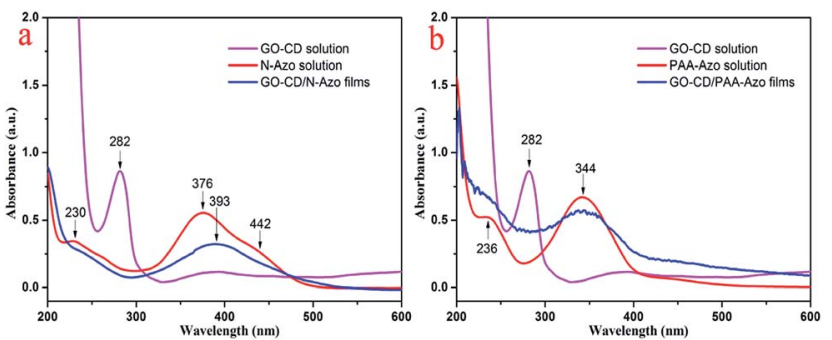

Fig. 4 UV-vis spectra of GO-CD solution and multilayer LB films of $\mathrm{GO}-\mathrm{CD} / \mathrm{N}-\mathrm{AzO}$ and GO-CD/PAA-Azo transferred at $15 \mathrm{mN} \mathrm{m}^{-1}$. 
azobenzene compounds were also measured and shown in Fig. S12 and S13. $\dagger$ No shift appeared for the mixed system of GO and N-Azo, indicating the adsorption process of N-Azo on GO surface in monodispersed state. However, for the mixed methanol solutions of GO-CD with N-Azo, the characteristic peak shifted from 376 to $384 \mathrm{~nm}$, suggesting formation of weak J-aggregate. While for the case of mixed solution of GO-CD with PAA-Azo, no obvious shift was observed.

In order to further confirm the interaction between GO-CD building blocks and azobenzene-containing molecules in the subphases, the FT-IR spectra of the obtained LB films were also measured. Obvious changes were observed for the IR spectra of prepared composite LB films after interacting with N-Azo and PAA-Azo molecules (Fig. 5a). In comparison with the multilayer LB films of GO-CD from water surface, the spectra of composite films show obvious intensity for bands at $3239 \mathrm{~cm}^{-1}(\mathrm{~N}-\mathrm{H}$ stretching and hydrogen bonding), 2923 and $2849 \mathrm{~cm}^{-1}(\mathrm{C}-\mathrm{H}$ modes of methylene moieties), $1718 \mathrm{~cm}^{-1}$ ( $\mathrm{C}=\mathrm{O}$ stretching), and $1636 \mathrm{~cm}^{-1}$ (amide $\mathrm{C}=\mathrm{O}$ stretching). In addition, the bands at 1454 and $1414 \mathrm{~cm}^{-1}$ can be assigned to the azobenzene groups, while the additional peaks at 1154,1082 and $1031 \mathrm{~cm}^{-1}$ can be assigned to the vibrations from beta-CD skeletons. At the same time, in comparison with the IR spectra of N-Azo and assynthesized PAA-Azo material in Fig. S10, $\uparrow$ the obvious changes for the LB films indicated the successful composition of two azobenzene-containing molecules with GO-CD materials in the organized self-assembly films.

It is well established that Raman spectroscopy has been widely used to investigate GO-based composite materials. The measurements for present as-obtained composite LB films of GO-CD from N-Azo and PAA-Azo subphase were performed. Three characteristic bands of graphene sheets in Raman spectra appear in Fig. 5b. One band at $1597 \mathrm{~cm}^{-1}$ can be owing to the $\mathrm{G}$ band originating from the first-order scattering of the $E_{2 g}$ phonons of the $\mathrm{sp}^{2}$-hybridized carbon atoms. Another band at
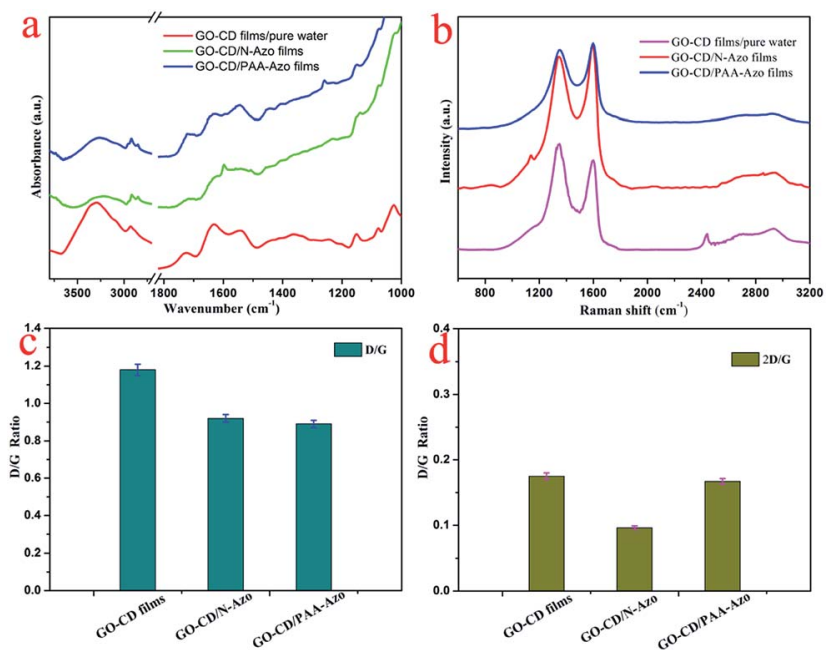

Fig. 5 IR spectra (a) and Raman spectra (b) of the multilayer LB films of GO-CD from water surface, N-Azo and PAA-Azo subphase transferred at $15 \mathrm{mN} \mathrm{m}^{-1}$. Pictures ( $c$ and d) indicate the $\mathrm{D} / \mathrm{G}$ ratio and $2 \mathrm{D} / \mathrm{G}$ ratio analysis in Raman spectra.
$1351 \mathrm{~cm}^{-1}$ can be assigned to the $\mathrm{D}$ band, which comes from a breathing mode of $\kappa$-point phonons of $\mathrm{A}_{1 \mathrm{~g}}$ symmetry of the defects involved in the $\mathrm{sp}^{3}$-hybridized carbon bonds such as hydroxyl and/or epoxide bonds. ${ }^{75,76}$ In addition to the $\mathrm{G}$ and $\mathrm{D}$ bands, a broad peak at $2690 \mathrm{~cm}^{-1}$ (i.e., 2D band) was also observed. The intensity of $2 \mathrm{D}$ band is correlated to the stacking mode of graphene sheets. It was well-known that the D/G peak intensity ratio could be utilized as a measurement of the $\mathrm{sp}^{2}$ domain size of graphene sheets containing $\mathrm{sp}^{3}$ and $\mathrm{sp}^{2}$ bonds due to the origination of $\mathrm{G}$ and $\mathrm{D}$ bands. ${ }^{77,78}$ For present system in Fig. 5c, the D/G ratio shifted from 1.18 for GO-CD LB films from water surface to 0.92 and 0.89 for the obtained $\mathrm{GO}-\mathrm{CD} /$ $\mathrm{N}-\mathrm{Azo}$ and GO-CD/PAA-Azo composite LB films. The obvious change confirmed the successful reaction of N-Azo molecules and polymeric PAA-Azo molecules with azobenzene groups in the formation process of composite films. Furthermore, the $2 \mathrm{D} / \mathrm{G}$ intensity ratios for the single and bilayer GO sheets were found in the range of 1.53-1.68 and $0.82-0.89$, respectively. ${ }^{79,80}$ Previous studies have also reported that the $2 \mathrm{D} / \mathrm{G}$ intensity ratios for single-, double-, triple-, and multi- $(>4)$ layer graphene sheets are 1.6, 0.8, 0.30 and 0.07 , respectively. Fig. $5 \mathrm{~d}$ shows that the $2 \mathrm{D} / \mathrm{G}$ ratios of the obtained LB films prepared in this study have values between 0.096 and 0.175 , further suggesting the multilayer nature in present GO-CD LB films.

To investigate the composition and chemical state of present prepared composite films, X-ray photoelectron spectroscopy (XPS) measurements were performed. The survey data for the obtained composite films show the characteristic $\mathrm{C}(1 \mathrm{~s}), \mathrm{O}(1 \mathrm{~s})$, and $\mathrm{N}(1 \mathrm{~s})$ peaks (Fig. 6). The deconvolution of $\mathrm{C}(1 \mathrm{~s})$ peaks from the multilayer LB films of GO-CD from pure water surface in Fig. 7a demonstrate the peaks centered at positions of 286.5, 288.4, and $289.9 \mathrm{eV}$, which can be assigned to the $\mathrm{C}-\mathrm{O}, \mathrm{C}=\mathrm{O}$, and $\mathrm{C}=\mathrm{O}-\mathrm{O}$ oxygen-containing bonds, respectively. ${ }^{81,82}$ For composite films GO-CD/N-Azo and GO-CD/PAA-Azo, the deconvolution of $\mathrm{C}(1 \mathrm{~s})$ peaks (Fig. $7 \mathrm{~d}$ and $\mathrm{g}$ ) display the peaks centered at positions of 284.8 and $285.4 \mathrm{eV}$ attributed to $\mathrm{C}-\mathrm{C}$, $\mathrm{C}=\mathrm{C} \& \mathrm{C}-\mathrm{H}$ as well as $\mathrm{C}-\mathrm{N}$ bonds. This confirmed the

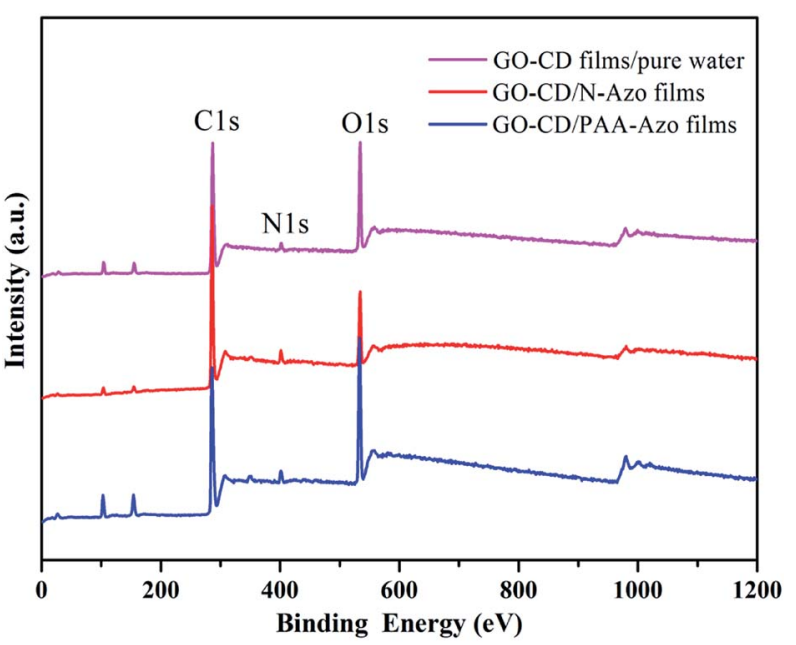

Fig. 6 Survey XPS spectra of the multilayer LB films of GO-CD from different subphases transferred at $15 \mathrm{mN} \mathrm{m}^{-1}$. 

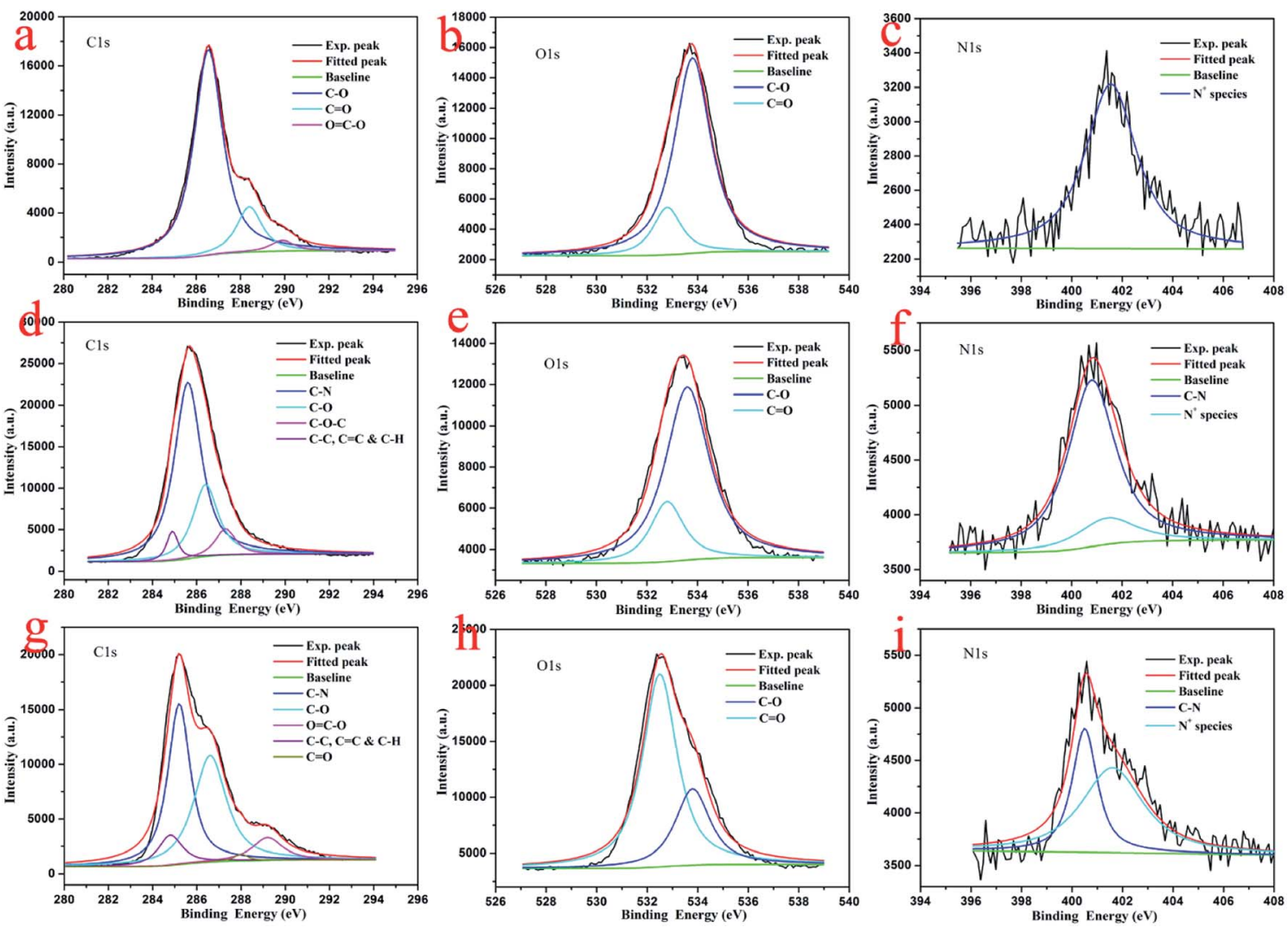

Fig. 7 Deconvolution of XPS peaks of multilayer LB films of GO-CD from pure water surface (a-c), GO-CD/N-Azo (d-f), and GO-CD/PAA-Azo (g-i) transferred at $15 \mathrm{mN} \mathrm{m}^{-1}$

successful hybrid of N-Azo and PAA-Azo molecules in composite films. In addition, the deconvolution of $\mathrm{O}(1 \mathrm{~s})$ peaks from the $\mathrm{LB}$ films from pure water surface in Fig. $7 \mathrm{~b}$ demonstrate the peaks centered at positions of 532.5 and $533.8 \mathrm{eV}$, which can be assigned to the $\mathrm{C}=\mathrm{O}$ and $\mathrm{C}-\mathrm{O}$ oxygen-containing bonds, respectively. However, the area ratio assigned to $\mathrm{C}=\mathrm{O}$ bond in $\mathrm{O}(1 \mathrm{~s})$ peak (Fig. 7h) from GO-CD/PAA-Azo composite films show the value of $72 \%$ in comparison with the value of $16 \%$ in GO-CD films from pure water surface, indicating the increment of carboxyl groups and addition of PAA-Azo molecules in the obtained composite LB films. Moreover, the deconvolution analysis of $\mathrm{N}(1 \mathrm{~s})$ peaks from the LB films of GO-CD from pure water surface in Fig. $7 \mathrm{c}$ demonstrate the single peak centered at positions of $401.6 \mathrm{eV}$ assigned to the $\mathrm{N}^{+}$species from beta-CD segments in GO-CD building blocks. ${ }^{83}$ After interacting with N-Azo or PAA-Azo, the deconvolution of N(1s) peaks (Fig. $7 \mathrm{f}$ and i) show also the new peak at $400.5 \mathrm{eV}$ assigned to the $\mathrm{C}-\mathrm{N}$ bond, which indicate the successful addition of azobenzene part in composite films.

To investigate the photoresponsiveness of present azobenzene-containing compounds, the aqueous solutions and composite LB films were irradiated by a UV lamp with wavelength of $365 \mathrm{~nm}$, and the change of the configuration of the azobenzene units was monitored by UV-vis spectroscopy. Fig. 8a and b shows the UV-vis absorption spectra of the obtained aqueous solutions of two azobenzene compounds upon UV irradiation in different time. As for the case of N-Azo solution, the $\pi-\pi^{*}$ transition band of trans isomers with $\lambda_{\max }$ at $376 \mathrm{~nm}$ decreases slightly. After irradiation for $10 \mathrm{~min}$, the UV-vis spectra do not change any more, indicating azobenzene groups reach to a trans-cis equilibrium state. For the PAA-Azo solution, with time increment, the $\pi-\pi^{*}$ transition band of trans isomers with $\lambda_{\max }$ at $344 \mathrm{~nm}$ decreases remarkably and finally shift to $325 \mathrm{~nm}$, at the same time, the $\mathrm{n}-\pi^{*}$ transition band of cis isomers at around $438 \mathrm{~nm}$ increases slightly. After irradiation for $5 \mathrm{~min}$, the UV-vis spectra do not change any more, indicating azobenzene groups reach to a cis saturated state. In addition, the obtained GO-CD/N-Azo and GO-CD/PAA-Azo composite LB films were investigated upon irradiation with UV irradiation in different time. As shown in Fig. 8c, the obtained GO-CD/N-Azo composite LB films showed slight decrement at $393 \mathrm{~nm}$ even upon UV irradiation for $30 \mathrm{~min}$, suggesting possible large spacial hindrance for trans-cis photoisomerization due to the J-aggregates of azobenzene parts in LB films. Moreover, Fig. 8d demonstrates the obvious spectral change in GO-CD/PAA-Azo composite LB films with UV irradiation in different time. The obtained spectral changes suggested that there was no strong aggregation between azobenzene groups in GO-CD/PAA-Azo composite LB films. The present results were in well accordance with the above obtained results.

Considering the above results, the self-assembly modes and stacking change of all prepared composite Langmuir films at the air/water interface can be proposed, as schematically shown 

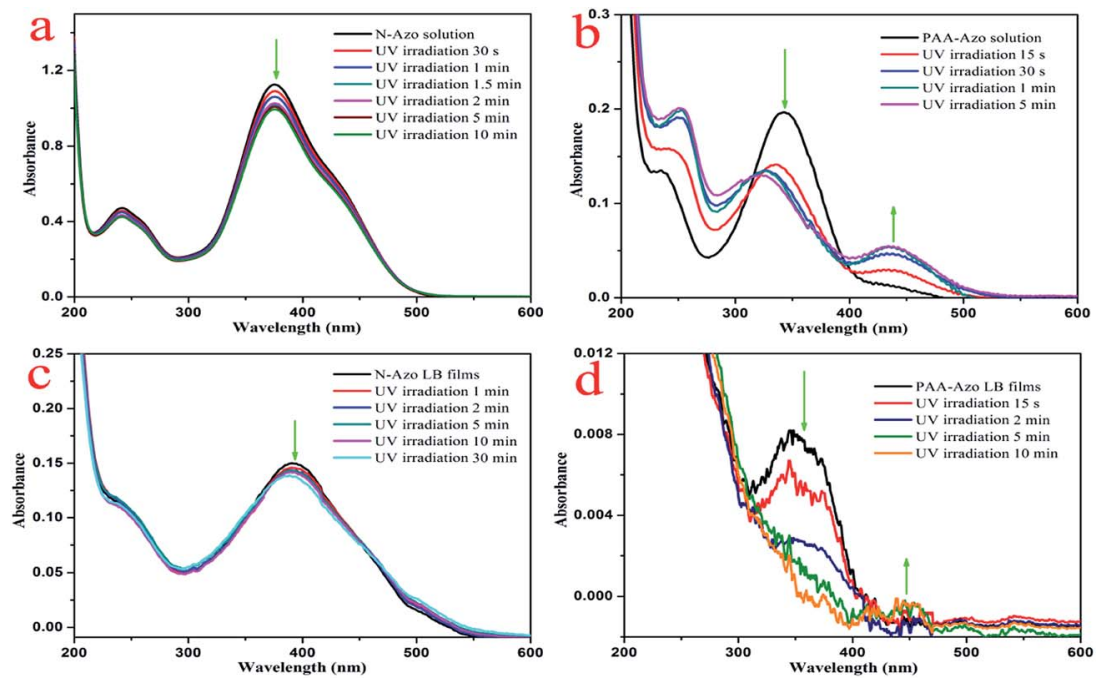

Fig. 8 UV-vis spectral changes of N-Azo and PAA-Azo solutions ( $a$ and b) as well as two composite LB films (c and d) upon UV irradiation at $365 \mathrm{~nm}$ for different time.

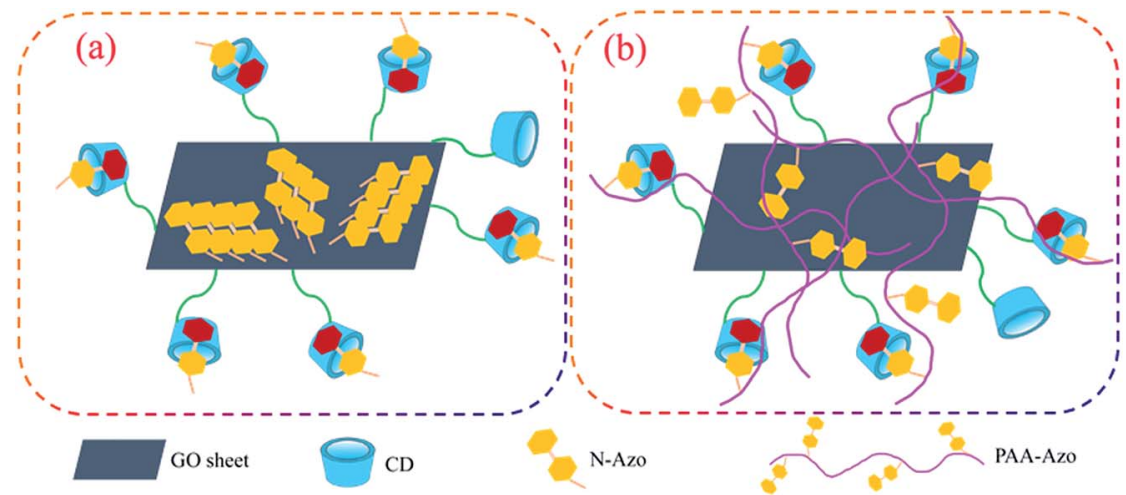

Fig. 9 Schematic illustration of reasonable self-assembly models in GO-CD/N-Azo (a) and GO-CD/PAA-Azo (b) composite Langmuir films.

in Fig. 9, which was in well accordance with our initial proposal to design this work. For the GO-CD/N-Azo composite Langmuir film, because of the hydrophilic amine moieties and aromatic azobenzene segments in N-Azo molecules, in the self-assembly process on water surface, the adjacent N-Azo molecules can be adsorbed on GO sheet surface and pack organized to overlap each other to form the J-aggregate domains (Fig. 9a). At the same time, some N-Azo molecules can penetrate into the beta$\mathrm{CD}$ cavity to form inclusion complex with dynamic equilibrium. ${ }^{84,85}$ This deduction was verified from the surface pressurearea isotherms, UV-vis spectra, and morphological characterization. As for GO-CD/PAA-Azo composite Langmuir film, the situation is greatly different. Azobenzene segments were linked with PAA long alkyl chains via amide bonds and well dispersed in the composite film. Some azobenzene part can form inclusion complex with beta-CD cavity, while others can be adsorbed on GO sheet surface or embedded in the PAA chain kinking with dispersed state (Fig. 9b). In this state, the limiting molecular areas in isotherm become similar to that from pure water surface and none of J-aggregate of azobenzene parts appeared.
Thus GO sheet cross-linked morphologies with surface of messy thread were observed. In addition, it should be noted that the CD spectra of the transferred multilayer $\mathrm{GO}-\mathrm{CD} / \mathrm{N}-\mathrm{Azo}$ and GO-CD/PAA-Azo composite LB films have been measured, as shown in Fig. S14. $\dagger$ No obvious CD signals could be observed. This result can be reasonably explained by the minor beta-CD component in the synthesized GO-CD building blocks and subsequent fewer formed beta-CD/azobenzene inclusion complex in the composite films. The follow-up research work can focus on the preparation of new GO-CD materials with nanoscale GO sheet (multiple carboxyl group sites) or poly-betaCD segment (multiple CD cavities) to improve the inclusion effect in the proposed LB films.

\section{Conclusions}

In summary, we have presented the design and characterization of beta-cyclodextrin-modified graphene oxide composite Langmuir films with some azobenzene compounds. The synthesized beta-cyclodextrin-modified graphene oxide GO-CD acts as 
either a host agent or a building block/amphiphile to form spreading films on different subphases. Depending on the different molecular structures of N-Azo and PAA-Azo molecules, variable self-assembly processes in the obtained composite films were achieved. When the building block GO-CD formed composite Langmuir films on N-Azo subphase, both host-guest interaction between beta-CD with azobenzene and J-aggregate of N-Azo molecules on GO sheet surface appeared. When GO-CD was spread on the subphase containing PAA-Azo, crosslinked nanostructures with messy thread and inclusion complex in composite films were obtained without any aggregates of azobenzene segment. Owing to the specific properties and self-assembled nanostructures in the obtained composite films, the present research work can demonstrate great potentials for the preparation of self-assembled nano-device films and nanosensors materials.

\section{Conflicts of interest}

There are no conflicts to declare.

\section{Acknowledgements}

This work was financially supported by the National Natural Science Foundation of China (No. 21473153), Support Program for the Top Young Talents of Hebei Province, China Postdoctoral Science Foundation (No. 2015M580214), and Scientific and Technological Research and Development Program of Qinhuangdao City (No. 201701B004 and 201502A006).

\section{Notes and references}

1 R. Narayan, J. E. Kim, J. Y. Kim, K. E. Lee and S. O. Kim, Adv. Mater., 2016, 28, 3045-3068.

2 Y. Y. Zhang, S. S. Gong, Q. Zhang, P. Ming, S. J. Wan, J. S. Peng, L. Jiang and Q. F. Cheng, Chem. Soc. Rev., 2016, 45, 2378-2395.

3 F. Li, X. Jiang, J. J. Zhao and S. B. Zhang, Nano Energy, 2015, 16, 488-515.

4 M. Daud, M. S. Kamal, F. Shehzad and M. A. Al-Harthi, Carbon, 2016, 104, 241-252.

5 G. H. Jeong, S. Baek, S. Lee and S. W. Kim, Chem.-Asian. J., 2016, 11, 949-964.

6 T. Jiao, Y. Liu, Y. Wu, Q. Zhang, X. Yan, F. Gao, A. Bauer, J. Liu, T. Zeng and B. Li, Sci. Rep., 2015, 5, 12451.

7 J. Texter, Curr. Opin. Colloid Interface Sci., 2015, 20, 454-464. 8 S. Gambhir, R. Jalili, D. L. Officer and G. G. Wallace, NPG Asia Mater., 2015, 7, e186.

9 R. Xing, T. Jiao, Y. Liu, K. Ma, Q. Zou, G. Ma and X. Yan, Polymers, 2016, 8, 181.

10 S. Niyogi, E. Bekyarova, M. E. Itkis, J. L. Mcwilliams, M. A. Hamon and R. C. Haddon, J. Am. Chem. Soc., 2006, 128, 7720-7721.

11 S. Stankovich, R. D. Piner, S. B. T. Nguyen and R. S. Ruoff, Carbon, 2006, 44, 3342-3347.

12 Z. B. Liu, Y. F. Xu, X. Y. Zhang, X. L. Zhang, Y. S. Chen and J. G. Tian, J. Phys. Chem. B, 2009, 113, 9681-9686.
13 I. Kaminska, A. Barras, Y. Coffinier, W. Lisowski, J. Niedziolka-Jonsson, P. Woisel, J. Lyskawa, M. Opallo, A. Siriwardena, R. Boukherroub and S. Szunerits, ACS Appl. Mater. Interfaces, 2012, 4, 5386-5393.

14 I. Kaminska, M. R. Das, Y. Coffinier, J. Niedziolka-Jonsson, J. Sobczak, P. Woisel, J. Lyskawa, M. Opallo, R. Boukherroub and S. Szunerits, ACS Appl. Mater. Interfaces, 2012, 4, 1016-1020.

15 Y. F. Yang, J. Wang, J. Zhang, J. C. Liu, X. L. Yang and H. Y. Zhao, Langmuir, 2009, 25, 11808-11814.

16 H. J. Salavagione, M. A. Gómez and G. Martínez, Macromolecules, 2009, 42, 6331-6334.

17 A. F. Hirschbiel, B. V. K. J. Schmidt, P. Krolla-Sidenstein, J. P. Blinco and C. Bamer-Kowollik, Macromolecules, 2015, 48, 4410-4420.

18 L. Peng, H. J. Zhang, A. C. Feng, M. Huo, Z. L. Wang, J. Hu, W. P. Gao and J. Y. Yuan, Polym. Chem., 2015, 6, 3652-3659.

19 S. B. Xie, J. Zhang, Y. L. Yuan, Y. Q. Chai and R. Yuan, Chem. Commun., 2015, 51, 3387-3390.

20 Y. L. Wu, R. F. Shi, Y. L. Wu, J. M. Holcroft, Z. C. Liu, M. Frasconi, M. R. Wasielewski, H. Li and J. F. Stoddart, J. Am. Chem. Soc., 2015, 137, 4111-4118.

21 H. Zhao, L. Yang, Y. Li, X. Ran, H. Ye, G. Zhao, Y. Zhang, F. Liu and C. P. Li, Biosens. Bioelectron., 2017, 89, 361-369.

22 L. Yang, H. Zhao, Y. Li, Y. Zhang, H. Ye, G. Zhao, X. Ran, F. Liu and C. P. Li, Biosens. Bioelectron., 2017, 87, 737-744.

23 L. Yang, S. Fan, G. Deng, Y. Li, X. Ran, H. Zhao and C. P. Li, Biosens. Bioelectron., 2015, 68, 617-625.

24 L. Yang, H. Zhao, C. P. Li, S. Fan and B. Li, Biosens. Bioelectron., 2015, 64, 126-130.

25 L. Yang, H. Zhao, Y. Li and C. P. Li, Sens. Actuators, B, 2015, 207, 1-8.

26 X. Ran, L. Yang, J. Zhang, G. Deng, Y. Li, X. Xie, H. Zhao and C. P. Li, Anal. Chim. Acta, 2015, 892, 85-94.

27 L. Yang, H. Zhao, S. Fan, G. Zhao, X. Ran and C. P. Li, RSC Adv., 2015, 5, 64146-64155.

28 X. Ran, L. Yang, G. Zhao, H. Ye, Y. Zhang, S. Fan, X. Xie, H. Zhao and C. P. Li, RSC Adv., 2015, 5, 60775-60785.

29 H. Yan, L. L. Zhu, X. Li, A. Kwok, X. Y. Pan and Y. L. Zhao, Asian J. Org. Chem., 2012, 1, 314-318.

30 Q. Xue, Z. G. Liu, Y. J. Guo and S. J. Guo, Biosens. Bioelectron., 2015, 68, 429-436.

31 A. Abbadessa, M. M. Blokzijl, V. H. M. Mouser, P. Marica, J. Malda, W. E. Hennink and T. Vermonden, Carbohydr. Polym., 2016, 149, 163-174.

32 T. Wang, N. Wu, H. Li, Q. L. Lu and Y. Jiang, J. Appl. Polym. Sci., 2016, 133, 43836.

33 H. Cao, X. Zhu and M. Liu, Angew. Chem., Int. Ed. Engl., 2013, 52, 4122-4126.

34 M. Deng, L. Zhang, Y. Jiang and M. Liu, Angew. Chem., Int. Ed. Engl., 2016, 55, 15062-15066.

35 P. Guo, L. Zhang and M. Liu, Adv. Mater., 2006, 18, 177-180. 36 X. Huang, C. Li, S. G. Jiang, X. S. Wang, B. W. Zhang and M. H. Liu, J. Am. Chem. Soc., 2004, 126, 1322-1323.

37 J. Jiang, Y. Meng, L. Zhang and M. Liu, J. Am. Chem. Soc., 2016, 138, 15629-15635. 
38 C. Liu, D. Yang, Q. Jin, L. Zhang and M. Liu, Adv. Mater., 2016, 28, 1644-1649.

39 L. J. Cote, F. Kim and J. Huang, J. Am. Chem. Soc., 2009, 131, 1043-1049.

40 F. Kim, L. J. Cote and J. Huang, Adv. Mater., 2010, 22, 19541958.

41 K. L. Harrison, L. B. Biedermann and K. R. Zavadil, Langmuir, 2015, 31, 9825-9832.

42 M. M. Jaafar, G. P. M. K. Ciniciato, S. A. Ibrahim, S. M. Phang, K. Yunus, A. C. Fisher, M. Iwamoto and P. Vengadesh, Langmuir, 2015, 31, 10426-10434.

43 F. Han, S. M. Yang, W. X. Jing, Z. D. Jiang, H. Liu and L. Li, Appl. Surf. Sci., 2015, 345, 18-23.

44 T. H. Han, J. Nanosci. Nanotechnol., 2015, 15, 1191-1194.

45 J. D. Mangadlao, C. M. Santos, M. J. L. Felipe, A. C. C. de Leon, D. F. Rodrigues and R. C. Advincula, Chem. Commun., 2015, 51, 2886-2889.

46 B. Gur, M. Sinoforoglu and K. Meral, RSC Adv., 2015, 5, 552557.

47 B. T. McGrail, J. D. Mangadlao, B. J. Rodier, J. Swisher, R. Advincula and E. Pentzer, Chem. Commun., 2016, 52, 288-291.

48 J. Wen, Y. Jiang, Y. Yang and S. Li, J. Mater. Sci.: Mater. Electron., 2014, 25, 063-1071.

49 P. F. Duan, L. Qin and M. H. Liu, Langmuir, 2011, 27, 13261331.

50 S. J. Li, D. Taura, A. Hashidzume and A. Harada, Chem.-Asian J., 2010, 5, 2281-2289.

51 Y. G. Li and M. H. Liu, J. Colloid Interface Sci., 2007, 306, 386390.

52 E. Rivera, M. D. P. Carreon-Castro, L. Rodriguez, G. Cedillo, S. Fomine and O. G. Morales-Saavedra, Dyes Pigm., 2007, 74, 396-403.

53 Y. Matsuzawa, S. Noguchi, H. Sakai, M. Abe and M. Matsumoto, Thin Solid Films, 2006, 510, 292-296.

54 D. Li, M. B. Muller, S. Gilje, R. B. Kaner and G. G. Wallace, Nat. Nanotechnol., 2008, 3, 101-105.

55 S. J. Jeon, S. Y. Kwak, D. Yim, J. M. Ju and J. H. Kim, J. Am. Chem. Soc., 2014, 136, 10842-10845.

56 X. N. Luo, K. Ma, T. F. Jiao, R. R. Xing, L. X. Zhang, J. X. Zhou and B. B. Li, Nanoscale Res. Lett., 2017, 12, 99.

57 C. Y. Quan, J. X. Chen, H. Y. Wang, C. Li, C. Chang, X. Z. Zhang and R. X. Zhuo, ACS Nano, 2010, 4, 4211-4219.

58 W. Tang and S. C. Ng, Nat. Protoc., 2008, 3, 691-697.

59 E. S. Orth, J. E. S. Fonsaca, S. H. Domingues, H. Mehl, M. M. Oliveira and A. J. G. Zarbin, Carbon, 2013, 61, 543-550.

60 G. Chen, S. Zhai, Y. Zhai, K. Zhang, Q. Yue, L. Wang, J. Zhao, H. Wang, J. Liu and J. Jia, Biosens. Bioelectron., 2011, 26, 3136-3141.
61 M. Liu, L. Zhang and T. Wang, Chem. Rev., 2015, 115, 73047397.

62 Y. Liu, T. Wang, Y. Huan, Z. Li, G. He and M. Liu, Adv. Mater., 2013, 25, 5875-5879.

63 Z. Shen, T. Wang and M. Liu, Angew. Chem., Int. Ed. Engl., 2014, 53, 13424-13428.

64 J. Yuan and M. Liu, J. Am. Chem. Soc., 2003, 125, 5051-5056.

65 L. Zhang, Q. Lu and M. Liu, J. Phys. Chem. B, 2003, 107, 25652569.

66 Q. Bao, D. Zhang and P. Qi, J. Colloid Interface Sci., 2011, 360, 463-470.

67 R. Zhang, R. Xing, T. Jiao, K. Ma, C. Chen, G. Ma and X. Yan, ACS Appl. Mater. Interfaces, 2016, 8, 13262-13269.

68 X. Zhao, K. Ma, T. Jiao, R. Xing, X. Ma, J. Hu, H. Huang, L. Zhang and X. Yan, Sci. Rep., 2017, 7, 44076.

69 Y. Liu, K. Ma, T. Jiao, R. Xing, G. Shen and X. Yan, Sci. Rep., 2017, 7, 42978.

70 R. Xing, W. Wang, T. Jiao, K. Ma, Q. Zhang, W. Hong, H. Qiu, J. Zhou, L. Zhang and Q. Peng, ACS Sustainable Chem. Eng., 2017, 5, 4948-4956.

71 F. Xie, G. Ouyang, L. Qin and M. Liu, Chem.-Eur. J., 2016, 22, 18208-18214.

72 R. Xing, K. Liu, T. Jiao, N. Zhang, K. Ma, R. Zhang, Q. Zou, G. Ma and X. Yan, Adv. Mater., 2016, 28, 3669-3676.

73 S. Huo, P. Duan, T. Jiao, Q. Peng and M. Liu, Angew. Chem., Int. Ed., 2017, DOI: 10.1002/anie.201706308.

74 E. D. Grayfer, A. S. Nazarov, V. G. Makotchenko, S. J. Kim and V. E. Fedorov, J. Mater. Chem., 2011, 21, 3410-3414.

75 T. F. Jiao, Y. J. Wang, F. Q. Gao, J. X. Zhou and F. M. Gao, Prog. Nat. Sci.: Mater. Int., 2012, 22, 64-70.

76 A. C. Ferrari and J. Robertson, Phys. Rev. B, 2000, 61, 1409514107.

77 L. M. Malard, M. A. Pimenta, G. Dresselhaus and M. S. Dresselhaus, Phys. Rep., 2009, 473, 51-87.

78 K. S. Kim, Y. Zhao, H. Jang, S. Y. Lee, J. M. Kim, K. S. Kim, J. H. Ahn, P. Kim, J. Y. Choi and B. H. Hong, Nature, 2009, 457, 706-710.

79 K. N. Kudin, B. Ozbas, H. C. Schniepp, R. K. Prudhomme, I. A. Aksay and R. Car, Nano Lett., 2008, 8, 36-41.

80 O. Akhavan, Carbon, 2015, 81, 158-166.

81 H. R. Thomas, A. J. Marsden, M. Walker, N. R. Wilson and J. P. Rourke, Angew. Chem., Int. Ed., 2014, 53, 7613-7618.

82 X. Chen, Y. C. Dai, Z. B. Zheng and K. Z. Wang, J. Colloid Interface Sci., 2013, 402, 107-113.

83 H. Guo, T. Jiao, Q. Zhang, W. Guo, Q. Peng and X. Yan, Nanoscale Res. Lett., 2015, 10, 272.

84 Z. Z. Tong, R. Y. Wang, J. Huang, J. T. Xu and Z. Q. Fan, Polym. Chem., 2015, 6, 2214-2225.

85 Q. Jin, G. Liu, X. Liu and J. Ji, Soft Matter, 2010, 6, 5589-5595. 